\title{
EVALUATION OF PEDIATRIC RHEUMATOLOGY KNOWLEDGE OF PEDIATRICIANS IN THE HEALTH CARE SERVICE OF THE CITY OF SÃO CAETANO DO SUL - SP
}

\author{
Giovanna Gotardini Baptista1,*, Vanessa Bugni Miotto e Silva ${ }^{1}$ \\ 1. Universidade Municipal de São Caetano do Sul, São Caetano do Sul (SP), Brazil. \\ *Corresponding author: gigi_baptista28@hotmail.com
}

\section{BACKGROUND}

Failure to recognize the signs and symptoms of pediatric rheumatic diseases can lead to delays in its diagnosis and treatment. In this context, it is essential for pediatricians to identify and refer suspected cases properly, avoiding morbidity and mortality, improving the quality of life of patients, and reducing costs to the health system. The aim of this study was to evaluate the knowledge in pediatric rheumatology of pediatricians in the health care service of São Caetano do Sul/SP.

\section{MATERIALS AND METHODS}

Two questionnaires were applied to the pediatricians. The first questionnaire was about demographic data (gender, age, year and undergraduate institution, complementary training in residency and/or practice area in pediatrics, length of experience, previous contact with rheumatology in training and/or medical practice). The second questionnaire contained 20 questions related to knowledge about pediatric rheumatology (history and physical examination, warning signs, musculoskeletal pain, differential diagnosis of acute arthritis, juvenile idiopathic arthritis (JIA) and common childhood vasculitis).

\section{RESULTS}

A total of 45 pediatricians answered the questionnaires, 28 (62.2\%) of whom were female, with a mean age of 38.9 years. The average time of experience was 11.7 years. Thirty-one (68.9\%) physicians studied at a private institution, 42 (93.3\%) completed medical residency in pediatrics, and 24 (53.3\%) also completed a pediatric specialty. The majority (93.3\%) reported previous contact with content in rheumatology, and 40 (88.9\%) had already accompanied a patient with rheumatic disease. The average number of correct answers in the questionnaire was 7.69, with a median of 7 . The maximum correct rate was $51.1 \%$ for questions about the differential diagnosis of acute arthritis and the minimum correct rate was $26.1 \%$ for questions related to JIA. There were no differences between the group with a questionnaire score greater than or equal to 7 and the group with a score less than 7.

\section{CONCLUSION}

This study pointed out that most of the evaluated pediatricians do not have adequate knowledge to recognize patients with pediatric rheumatic disease. There is a need for further discussion about these diseases, both in undergraduate and residency programs in pediatrics, so that the diagnosis and treatment of these diseases occur properly, contributing to better quality of life for these patients. 